\title{
The contribution of plutinos to the Centaur population
}

\author{
Romina P. Di Sisto, Adrián Brunini, and Gonzalo C. de Elía \\ Facultad de Ciencias Astronómicas y Geofísicas - UNLP, \\ IALP - CONICET, Paseo del Bosque $S / N$, La Plata. Argentina \\ email: romina@fcaglp.unlp.edu.ar, gdeelia@fcaglp.unlp.edu.ar, \\ abrunini@f caglp.unlp.edu.ar
}

\begin{abstract}
We present a study of the dynamical evolution of plutinos recently escaped from the resonance through numerical simulations. It was shown in previous works the existence of weakly chaotic orbits in the plutino population that diffuse very slowly finally diving into a strong chaotic region. These orbits correspond to long-term plutino escapers and then represent the plutinos that are escaping from the resonance at present. Then, we divided the numerical simulation in two parts. First, we develop a numerical simulation of 20,000 test particles in the resonance in order to detect the long-term escapers. We set the initial orbital elements such that cover the present observational range of orbital elements of plutinos. Second, we perform a numerical simulation of the selected escaped plutinos in order to study their dynamical post escaped behavior. We describe and characterize the routes of escape of plutinos and their evolution in the Centaur zone. Also, we obtain that Centaurs coming from plutinos would represent a fraction of less than $6 \%$ of the total Centaur population.
\end{abstract}

Keywords. Kuiper Belt, methods: numerical.

\section{Introduction}

Plutinos are the resonant transneptunian population most densely populated. They are trapped into the 2:3 mean motion resonance with Neptune, being Pluto its most representative member. They are in a stable configuration, with the critical angle $\sigma=3 \lambda-2 \lambda_{N}-\varpi$ librating around $180^{\circ}$, where $\lambda$ and $\varpi$ are the mean longitude and the longitude of perihelion of the particle, and $\lambda_{N}$ is the mean longitude of Neptune. Duncan et al. (1995), showed that the boundaries of the 2:3 long-lived mean motion resonance have a time scale for instability of the order of the age of the Solar System and they may be related to the origin of the observed JFCs. Morbidelli (1997) studied the dynamical structure of the 2:3 resonance in order to analyze possible diffusive phenomena and their relation to the existence of long-term escape trajectories. From his work it could be deduced a present rate of escape of 1 plutino with $R>1 \mathrm{~km}$ every 20 years. Yu \& Tremaine (1999) and Nesvorný et al. (2000) analyzed the effect of Pluto on the 2:3 resonant orbits. The latest found similar values for the escape rate as the ones obtained by Morbidelly (1997) without Pluto. Melita \& Brunini (2000) suggested that the existence of plutinos in very unstable regions can be explained by physical collisions or gravitational encounters with other plutinos. Nesvorný \& Roig (2001) explored the dynamics of the 2:3 resonance and showed that the regions of orbital stability do not seem to be well sampled by observed plutinos, which may be a dynamically primordial feature or a consequence of collisions and mutual gravitational scattering. De Elía et al. (2008) performed a collisional evolution of plutinos and obtained a plutino removal by their collisional evolution of 2 plutinos with $R>1 \mathrm{~km}$ every 10000 years. Since, from that previous works, plutino removal by "dynamic" is much greater than plutino removal by collisions we performe "dynamical" 
numerical simulations. We refer to "dynamical" numerical simulations, those that take into account the gravitational forces to follow up the evolution of a particle ocasionally causing the remotion. Our goal is to describe and characterize the routes of escape of plutinos and their contribution to the Centaur population.

\section{The numerical runs}

We need to identify the plutinos that have recently escaped from the resonance. Morbidelli (1997) showed the existence of weakly chaotic orbits that diffuse very slowly finally diving into a strong chaotic region. These orbits corresponds then to long-term escapers i.e. plutinos recently escaped from the resonance. Then we divided the numerical simulation in two parts. First: the pre-runs where we developed numerical simulations of plutinos in the resonance in order to detect those that escape at present. Second: the post-runs where we performed a numerical simulation of the selected escaped plutinos in order to study their dynamical post escaped behavior.

\subsection{Pre-runs. The integration in the resonance}

We performed a numerical integration following the study of Morbidelli (1997). We integrated here 20,000 test particles under the gravitational influence of the Sun and the four giant planets over 4.5 Gy with an integration step of 0.5 years using EVORB (Fernández et al. (2002)). We set the initial semimajor axis of the particles equal to the exact value of the resonance $a_{i}=39.5 \mathrm{AU}$. The initial argument of perihelion $\omega_{i}$, longitude of node $\Omega_{i}$ and the mean anomaly $M_{i}$ have been chosen at random in the range of $\left[0^{\circ}, 360^{\circ}\right]$ so the critical angle $\sigma$ remains between $180^{\circ}$ and $330^{\circ}$. Since $\sigma$ librates around $180^{\circ}$, and given the relation between $a$ and $\sigma$ in the resonance, the election of the previous orbital elements covers the 2:3 mean motion resonance (see Morbidelli (1997) for a complete explanation). The initial eccentricity and inclination of the particles have been randomly chosen in the intervals $[0,0.35]$ and $\left[0^{\circ}, 45^{\circ}\right]$, respectively, covering the present observational ranges for the orbital elements of Plutinos. The test particles where integrated up to the first encounter within the Hill sphere of a giant planet, collision onto a planet or ejection. Such final states represent the escape conditions of plutinos. So we can calculate the rate of escape of particles from the resonance.

In Fig. 1 we plot the cummulative number of escape particles from the resonance $\left(N_{e}\right)$ with respect to the number of the remaining particles $N_{s}$, where $N_{s}=20000-N_{e}$, as a function of $t$. It can be seen that the number of escaped particles raise quickly at the begining up to $t \sim 1.5 \mathrm{~Gy}$. In this point the slope of the curve changes and behaves roughly as a linear relation. This change of slope was already noticed by Morbildelli (1997) and is related with the time when the strongly chaotic region is completely depleted and

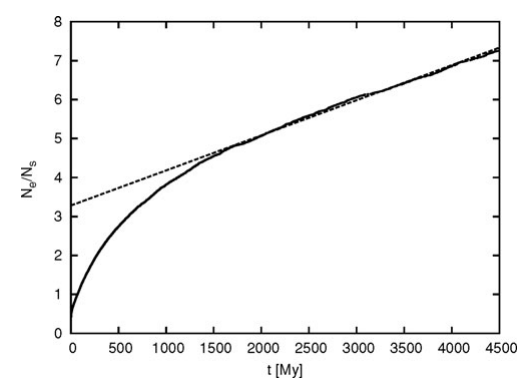

Figure 1. Cummulative number of escape particles from the resonance $\left(N_{e}\right)$ with respect to the number of the remaining particles $N_{s}$ versus time. 
the weakly chaotic region starts to be the dominant source of Neptune-encountering bodies. We fit to the plot for $t>1.5 \mathrm{~Gy}$, a linear relation $N_{e} / N_{s}=a t+b$ where: $a=9.00713 \times 10^{-10} \pm 4.735 \times 10^{-13} \mathrm{yr}^{-1}$ and $b=3.28079 \pm 0.001347$. The slope of the linear relation, $a$, represents the present rate of escape of the particles from the 2:3 mean motion resonance.

From our initial particles, we have 1183 that escape from the resonance after $t=1.5$ Gy. Since those particles would represent the present escaped plutinos, we identify their original orbital elements for the second integration.

\subsection{Post-runs. The Post escape integration.}

We numerically integrated again from $t=0$, the 1183 particles that escape at $t>1.5$ Gy, in order now, to obtain their post escape evolution. We integrated those particles with the same computing conditions than the previous integration but now we followed the integration for $10 \mathrm{~Gy}$. The particles were removed from the simulation when they either collide with a planet or the Sun, or they reached a semimajor axis greater than 1000 AU or they enter the region inside Jupiter's orbit $(r<5.2$ AU) where the perturbations of the terrestrial planets are not negligible. We recorded the orbital elements of the particles and the planets every $10^{4}$ years.

\section{Results}

In Fig. 2 we plot the time-weigthed distribution of escaped plutinos in the orbital element space. These plots assume time-invariability, so they don't represent the real case where plutinos are continously entering and leavig the zone, but they help to identify the densest and empties regions. The great majority of plutinos have encounters with Neptune, so this planet is the main responsible for their post escape evolution. This behavior can be seen in the plots as the densest zone near Neptune's perihelion. The densest zone in the other orbital elements of escaped plutinos corresponds to the ranges $30<a<100 \mathrm{AU}$ and $5^{\circ}<i<40^{\circ}$. When escaped plutinos are transferred to the SD (this is the zone of perihelion distances $q<30 \mathrm{AU}$ ) they are quickly locked into a mean motion resonance with Neptune (similar to the behavior of SDOs analized by Fernández et al. (2004) and Gallardo (2006)). In the Centaur zone (this is the zone of $q<30 \mathrm{AU}$ ) the distribution of escaped plutinos is similar to that of SDOs in the Centaur zone obtained by Di Sisto and Brunini (2007).

The escaped plutinos have a mean lifetime in the Centaur zone of $l_{C}=108 \mathrm{My}$, greater than that of Centaurs from SD of $l_{C}=72 \mathrm{My}$. Escaped-plutinos live more time than SDOs in the greater-perihelion Centaur zone, causing a slower diffusion to the inner Solar System of escaped-plutino orbits than of SDOs orbits.
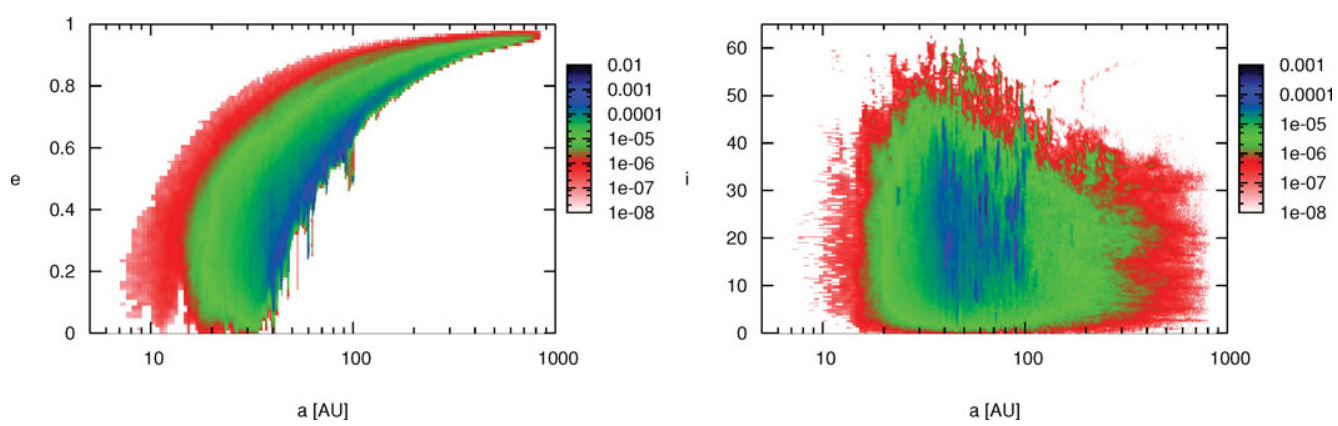

Figure 2. Time-weighted distribution of escaped plutinos in the orbital element space. 
In order to calculate the number of escaped plutinos located at present in the Centaur population, we calculate the present rate of injection of escaped plutinos into the Centaur zone. As we have mentioned we consider that the long-term escapers from the plutino population, i.e., those that escape after $t=1.5 \mathrm{~Gy}$, represent the present plutino espapers. Then the plot of the cummulative number of escaped plutinos injected into the Centaur zone $\left(N_{c}\right)$ with respect to the number of the remaining plutinos $N_{p}$, as a function of time is well fitted by the linear relation: $\frac{N_{c}}{N_{p}}=c t+d$, where $c=1.62076 \times 10^{-10} \pm 8.502 \times$ $10^{-14} \mathrm{yr}^{-1}$ and $b=-0.228442 \pm 0.000242$. The slope of the linear relation, $c$, represents the present rate of injection of plutinos into the Centaur zone. Assuming that the present number of plutinos, $N_{p}$, is constant with time, we can calculate the present number of escaped plutinos in the Centaur population as $N_{c}=c N_{p} l_{C}$ where $l_{C}$ is expressed in years. The number of small objects in the plutino population is not well determined, since the present observational surveys can't cover all the small-sized objects. The size distribution of plutinos is calculated from different surveys up to a given size, typically up to $R \sim 30 \mathrm{~km}$. For objects with radius less than this size, the population could have a break (Kenyon et al. (2007), Bernstein et al. (2004), Elliot et al. (2005)). Considering the possible existence of a break and its magnitude, the number of plutinos $N_{p}(R>1 \mathrm{~km})$ could be between $10^{8}$ and $10^{9}$. Then the present number of plutino-Centaurs with $R>1$ $\mathrm{km}$ would be between $1.8 \times 10^{6}-1.8 \times 10^{7}$. Di Sisto and Brunini (2007) estimated $N_{C}(R>1 \mathrm{~km}) \sim 2.8 \times 10^{8}$ coming from the SD, then Centaurs coming from plutinos would represent a fraction of less than $6 \%$ of the total population being then a secondary source of Centaurs.

\section{References}

Bernstein, G. M., Trilling, D. E., Allen, R. L., et al. 2004, AJ, 128, 1364

de Elía, G. C. \& Brunini, A. 2008, A\&A, 490, 835

Di Sisto, R. P. \& Brunini, A. 2007, Icarus, 190, 224

Duncan, M. J., Levison, H. F., \& Budd, S. M. 1995, AJ, 110, 3073

Elliot, J. L., Kern, S. D., Clancy, K. B., et al. 2005, AJ, 129, 1117

Fernández, J. A., Gallardo, T., \& Brunini, A. 2002, Icarus, 159, 358

Fernández, J. A., Gallardo, T., \& Brunini, A. 2004, Icarus, 172, 372

Gallardo, T. 2006, Icarus, 184, 29.

Kenyon, S. J., Bromley, B. C., O'Brien, D. P., \& Davis, D. R. 2008, in: M. A. Barucci, H. Boehnhardt, D. Cruikshank, \& A. Morbidelli (eds.), The Solar System Beyond Neptune, (Tucson: University of Arizona Press), p. 293

Melita, M. D. \& Brunini, A. 2000, Icarus, 147, 205

Morbidelli, A. 1997, Icarus, 127, 1

Nesvorný, D., Roig, F., \& Ferraz-Mello, S. 2000, AJ, 119, 953

Nesvorný, D. \& Roig, F. 2000, Icarus, 148, 282

Yu, Q. \& Tremaine, S. 1999, AJ, 118, 1873 